EPiC Series in Computing
Volume 69, 2020, Pages 264-276
$\begin{gathered}\text { Proceedings of 35th International Confer- } \\ \text { ence on Computers and Their Applications }\end{gathered}$

\title{
Real-Time Learner Classification Using Cognitive Score
}

\author{
Avick Kumar Dey ${ }^{1}$, Bibek Poddar ${ }^{2}$, Pijush Kanti Dutta Pramanik ${ }^{1}$, Narayan \\ C Debnath ${ }^{3}$, Sultan Aljahdali ${ }^{4}$ and Prasenjit Choudhury ${ }^{1}$ \\ ${ }^{1}$ National Institute of Technology Durgapur, India \\ ${ }^{2}$ Curiosta Innovation Labs LLP, India \\ ${ }^{3}$ Eastern International University, Vietnam \\ ${ }^{4}$ Taif University, Saudi Arabia \\ iavickdey@gmail.com, bibekpoddar06@gmail.com, \\ pijushjld@yahoo.co.in, narayan.debnath@eiu.edu.vn, \\ aljahdali@tu.edu.sa, prasenjit0007@yahoo.co.in
}

\begin{abstract}
Recommending and providing suitable learning materials to the learners according to their cognitive ability is important for effective learning. Assessing the cognitive load of a learner while studying a learning material can be helpful in assessing his/her intelligence and knowledge adapting abilities. This paper presents a real-time assessment method of the intelligence of students according to their instant learning skills. The proposed system can read the brain waves of students of different age groups at the time of learning and classify their instant learning skills using the cognitive score. Based on this, the learners are suggested suitable learning materials which maintain the learner in an overall state of optimal learning. The main issues concerning this approach are constructing cognitive state estimators from a multimodal array of physiological sensors and assessing initial baseline values, as well as changes in the baseline. These issues are discussed in a data processing block-wise structure. Synchronization of different data streams and feature extraction and formation of a cognitive state metric by classification/clustering of the feature sets are done. The results demonstrate the efficiency of using cognitive score in RTLCS in the identification of instant learning abilities of learners.
\end{abstract}

Keywords: Learner classification, Personalised learning, EEG signal, Cognitive score 


\section{Introduction}

Presently, education needs to keep up with the rapid increase in knowledge, therefore, necessitating the possession of a variety of intellectual capabilities of the students. These intellectual assets include abilities for gaining knowledge, regulating and using knowledge, evaluating the quality and application of knowledge, etc. [1]. A paradigm shift from teacher-centric to student-centric learning is needed [2]. Hence, the process of learning needs to be designed to foster an active, synergetic, self-regulated, and self-oriented learning environment. The process of learning requires that students must possess selfregulation, which enhances the ability to reason. The scenario of passively gaining knowledge by students should now move to higher levels of active imbibing of knowledge.

A few of the traits required for success are the capability of the followings [3]:

- Critical thinking, analysis, and solving practical problems

- Finding, evaluating, and using suitable resources for learning

- Working with teams

- Effective verbal and written communication skills

- Using knowledge and intellect for learning on a continuous basis

In addition to these traits, Trilling and Hood [4] noted that diligence, creativity, cross-cultural sensitivity, and computing skills matter too.

In such a scenario, it is important to devise ways of knowing how well students are able to absorb what is taught to them. Recommending and providing suitable learning materials to the learners according to their cognitive ability is important for effective learning [5]. For this, it is crucial to assess and estimate the learning ability of the learner correctly. One such important method is the learner classification technique for developing online lessons to suit each individual learner. Essentially, this system offers a solution for the drawbacks of our traditional and e-learning education systems. In a traditional education system, students are always dependent on their teachers for every study-related matter. This trait suppresses the creative side of their personality, and they never get to know their strengths. The teachers are unaware of what actually goes on in class. They cannot, simultaneously, teach and monitor their classrooms. The teachers are unaware of the weaknesses of their students, and thus, they are unable to offer them personalized learning that suits their needs. E-learning, by using a digital teacher, can address these problems of traditional teaching systems [6]. But the main drawback of e-learning is that all the students or learners are treated as being at the same level of learning.

Using learner classification, in a personalized e-learning system, the students are differentiated according to their learning skills, behaviours and performances individually. Learner classification using cognitive score is driven by the learning behaviour of students and their performance based on time. A learner perceives, remembers, thinks, and solves problems. How do learners process experience and knowledge? Do they learn sequentially or randomly?

Learner classification answers all such questions. To identify the quick learners among students of different streams and ages, electrical bio-signals will be used. Any signal that is measured and monitored from an organism is often referred to as an electrical bio-signal [7]. The electrical bio-signals in the body are produced as a result of the electrical activities of the cells. The human body produces many electrical signals, and one of them is the electroencephalography (EEG) signal, a measurement of the electrical activity that occurs in the brain [8]. The brain consists of billions of cells, half of which 
are neurons, and the other half of which help and facilitate the activity of neurons. These neurons are densely interconnected via synapses, which act as gateways of inhibitory or excitatory activity.

\subsection{The Need for Learner Classification}

The current education system is mainly based on a generalized approach which evaluates learners and their progress. The teacher is the key player in learning. Students, hence, are always dependent on their teachers. The teacher is not only required to teach the lesson, but also evaluate the progress of the students. This is done by behavioural examination and by asking a few questions related to the lesson and then assessing the student's behavioural changes. Teachers often show favouritism. It is noted that a reason for a pupils' low self-esteem in academics is favouritism [9]. Achievement in academics is the degree of aptitude gained in education, which is generally viewed as the marks scored by students in evaluations on subjects by the schools [10]. It is suggested that for the elimination of bias, teachers need to follow the same approach for all students [11]. However, a certain amount of bias always creeps in. Therefore, the traditional approach of teaching falls short of evaluating the progress of the students.

But with the advent of e-learning and its rapid progress, the teacher becomes a centralized figure. There is no one-to-one interaction between students and the teacher; rather, this interaction is a digital one. Also, it must be noted that not every student has the same grasping ability - some grasp faster than the others. However, a big disadvantage of e-learning is that it treats every learner equally, and so the pace and evaluation methods are the same.

Every individual has a different way of processing information in the way it is obtained, sorted, and utilized. Learner classifier systems help to understand the skills for gathering information and to calculate the time required for learning. There is a need to develop a system which can study the behaviour of a teacher towards understanding the progress of student learning. This can be achieved by using proper tools and designing algorithms that can determine the student's progress in a scientific manner. Thus, the present study proposes cognitive studies for evaluating learning patterns using a system of sensors and data-driven algorithms to enhance the learner experience of the e-Learning system. A learner classification has several benefits, as listed in Figure 1.

\begin{tabular}{|c|c|}
\hline $\begin{array}{c}\text { Adaptive in nature } \\
\text { Ability to gather } \\
\text { information }\end{array}$ & $\begin{array}{c}\text { • Acclimatization to a changing environment is easy as in online learning. } \\
\text { feasible. }\end{array}$ \\
\hline $\begin{array}{c}\text { Identifies stochastic } \\
\text { learner }\end{array}$ & $\begin{array}{c}\text { - Stochastic learning is beneficial in large-scale or highly complex issues } \\
\text { where deterministic or comprehensive learning is difficult. }\end{array}$ \\
\hline $\begin{array}{c}\text { Classifies quick } \\
\text { learner }\end{array}$ & $\begin{array}{c}\text { Classification of quick learners in real-time is feasible during learning or } \\
\text { reading processes. }\end{array}$ \\
\hline $\begin{array}{c}\text { Identifies } \\
\text { concentration level }\end{array}$ & $\begin{array}{c}\text { - Identification of the concentration level of different learners of different } \\
\text { ages is feasible. Higher concentration leads to quicker learning. }\end{array}$ \\
\hline
\end{tabular}

Figure 1. Benefits of learner classification 


\subsection{Authors' Contribution}

The following are the contributions of this study:

a. A system is designed that collects the brainwaves, evaluates the instantaneous attention level of the learner, transfers them to an android app, and sends the data to the server.

b. We acquire the reading from the system, which monitors the attention readings of the different learners (which includes both at a masters and diploma level) on a continuous basis.

c. Parameters are proposed for the calculation of the overall cognitive load of the document.

d. The cognitive load is calculated using the proposed algorithm.

e. Using the feature data sets, a classification engine for recommending different levels of learners is proposed.

\subsection{Organisation of the Paper}

The rest of the paper is organised as follows. The preliminary theoretical background, required for this paper, is briefly explained in Section 2. Section 3 mentions a few similar works. Section 4 describes the system architecture and its functionality. Section 5 discusses the experimental results. Section 6 concludes the paper.

\section{Theoretical Background}

This section presents an overview of the involved concepts.

\subsection{Brainwaves}

All synaptic activities create small electrical impulses called post-synaptic potential which may be measured on the surface of an individual's head. While conducting psychophysical research in the mid1920s and attempting to record the brain's psychic energy, German neurologist Hans Berger recorded the earliest electroencephalogram (EEG) [2]. Since this landmark discovery, EEGs have been employed for providing important information on the functioning and the mental state of the brain, including diagnosing of sleep conditions, epilepsy, Alzheimer's, etc. EEG studies are continually being enhanced by newer technologies. Without any complicated technologies, measurement and analysis of brain waves are possible using a simple EEG device having a dry electrode attached to the head [12].

The activity of the brain is usually described by a brain wave combination. The brain wave patterns vary with the cognitive processing and consciousness levels of an individual. The dominance of a particular brain wave over other waves depends on what is being read at that instant. As an instance, faster brainwaves will show dominance when a familiar topic is being read, and there is no stress, which indicates quick learning. Brain wave balance is essential, without which the learner might face stress and neurological/physical health issues.

Five varieties of brain waves exist, as shown in Table 1.

From the categories, we find that Beta Waves are mostly generated in the learning-based task. Most of the recent studies have been on developing methods to quantify Beta waves [13] [14] in the learning task. There have been special noninvasive electrodes that are placed on the pre-frontal scalp to record and then analyse this waveform. The studies also focus on developing standard algorithms the calculate the instantaneous attention level of the learner in a learning state. The difficulty faced by the learner is also referred to as the cognitive load. Therefore, these formulate the base of the cognitive load 
theory. But the studies failed to properly quantify the cognitive load of the learner for a particular document or context.

Table 1: Frequency bands of brainwaves based on mental states [15]

\begin{tabular}{|l|l|l|}
\hline \multicolumn{1}{|c|}{$\begin{array}{c}\text { Brainwave } \\
\text { type }\end{array}$} & \multicolumn{1}{c|}{$\begin{array}{c}\text { Frequency } \\
\text { range }\end{array}$} & \multicolumn{1}{c|}{ Mental states and conditions } \\
\hline Delta & $0.1 \mathrm{~Hz}-3 \mathrm{~Hz}$ & Deep, dreamless sleep, non-rapid-eye-movement sleep, unconscious \\
\hline Theta & $4 \mathrm{~Hz}-7 \mathrm{~Hz}$ & Intuitive, creative, recall, fantasy, imaginary, dream \\
\hline Alpha & $8 \mathrm{~Hz}-12 \mathrm{~Hz}$ & Relaxed, but not drowsy, calm, conscious \\
\hline Low beta & $12 \mathrm{~Hz}-15 \mathrm{~Hz}$ & Relaxed yet focused, integrated \\
\hline Midrange beta & $16 \mathrm{~Hz}-20 \mathrm{~Hz}$ & Thinking, aware of self and surroundings \\
\hline High beta & $21 \mathrm{~Hz}-30 \mathrm{~Hz}$ & Alertness, agitation \\
\hline Gamma & $30 \mathrm{~Hz}-100 \mathrm{~Hz}$ & Cognition, information processing \\
\hline
\end{tabular}

\subsection{Cognitive Load}

Cognitive loading occurs during learning tasks. They could limit the ability of learners in absorbing new knowledge and creating memories in the long-term. Such loads, generally, are increased when stressful demands are made to the student, rendering the processing of information overly complicated.

Intrinsic cognitive load: Here, the demand on a student is created by the intrinsic nature of the learnt information. The imposed load on a student is a function of the complexity of the assigned task or presented ideas, including the student's ability to comprehend the new concepts.

Extraneous cognitive load: This load is created by the demands made by the instructor on students, or the assignments that they are required to do. Such loads are external to the learning assignment and are enhanced when teaching techniques are ineffective, causing unintentional confusion with misleading information, thus making an assignment more complicated than it actually is.

Cognitive load as a function of cognitive score and load time: The cognitive load is different for each student. Reducing the total cognitive load on the student improves the learning quality. In this paper, we analytically defined the overall cognitive load as a function of the cognitive score and the load time for the of the learner for the given document. The cognitive score defined here is the average of the overall attention data above the standard deviation high bar (often called an exceptional point). The time for which the learner had the cognitive score is the load time (in seconds). Thus, the feature of for our classification engine (i.e. cognitive score and load time) is calculated.

\section{Related Work}

Earlier studies have used physiological signals to detect quick learners. For example, Choppin [16] built a quick learner recognizer for students of various ages, and also investigated EEG signals to identify mind waves, in order to provide a means to express the learning skills. In addition, this study used neural networks to classify the EEG signals online and achieved an accurate classification rate of about $64 \%$ of new unseen samples using three emotion classes and limited training data sets. Musha et al. [17] studied EEG signals to read mind waves and derived cross-correlation coefficients between the EEG activities from people of different ages and computed a 'learner matrix' to transform these coefficients linearly into a four-element vector that corresponds with some basic learning times. The numbers in the vector denote how strong that particular learning skill is found in the EEG signals. Earlier studies have investigated the estimated subjective time using neural networks to categorize learning states based on EEG features with respect to time. This study reported that the average accuracy 
ranges from $54.5 \%$ to $67.7 \%$ for each of the four emotional states. Heraz et al. [18] established an agent to predict emotional states during learning. The best classification in the study was the accuracy of $82.27 \%$ for distinguishing eight emotional states, using $k$-nearest neighbours as a classifier and the amplitudes of four EEG components as features. Chanel et al. [19] reported an average accuracy of 63\% by using EEG time-frequency information like features and support vector machines (SVM) as a classifier to characterize EEG signals into three emotional states. Zhang and Lee [20] proposed an emotion understanding system that classified users' status in two emotional states with the accuracy of $73.0 \% \pm 0.33 \%$ during image viewing. This system employed asymmetrical characteristics at the frontal lobe as features and SVM as a classifier. Finally, Lin et al. [21] applied machine learning (ML) techniques to categorize EEG signals according to the subject's self-reported emotional states during music listening. They propose a framework for systematically seeking emotion-specific EEG features and exploring the accuracy of the classifiers. They applied SVM to classify four emotional states: confused, taking time to learn, learning at medium time, and learning quickly.

As indicated in the literature survey, there has been continuous research in the field of quick learner classification. Many systems have been proposed to perform dynamic adaptation by reading brain signals. BCI (Brain-Computer Interface) technology is not considered as part of a mobile educational system that can surround the environment with reading mental signals to automatically adapt the content.

\section{Methods and Materials}

The following sections outline the experimental setup, including the system architecture, workflow, and the classification engine description.

\subsection{System Architecture}

The architecture of the proposed system consists of three components (Figure 2). The first component collects data from the EEG sensor through the Android application. The second component trains the system. The system establishes the relationship between EEG signals and learner attention. This is done automatically by the training component. This process needs to be run only once, after which the system will be able to recognize a quick learner from new EEG signals. The third component runs the system in a normal manner. This component does the same preprocessing and feature extraction steps as the training component. After the feature extraction, it uses the relationships extracted in the training phase to classify the EEG signals for quick learner classification. 


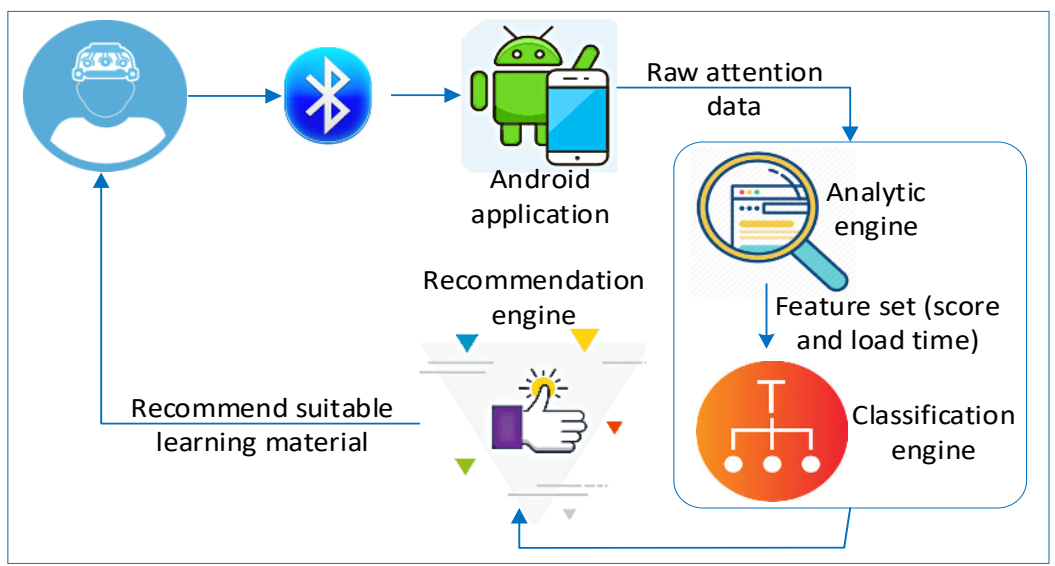

Figure 2. Proposed system architecture

\subsection{Workflow}

First, the new user or learner reads a document wearing the EEG sensor device. The learner needs to read the extract of both difficulty levels. Next, the mind waves or EEG signal data of the learner will be collected from the sensor via Bluetooth, following which the classification engine classifies the learner's EEG signal data according to the training datasets, and categorizes the learner between Beginner and Expert levels (Figure 3).

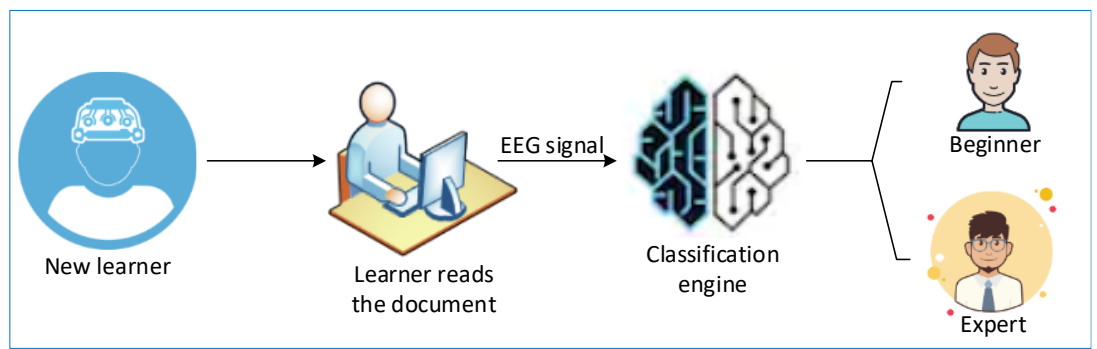

Figure 3. The workflow of the proposed system

\subsection{Classification Engine}

The proposed real-time classification engine works on six different components. First, the raw data are collected from the Android application, and it is converted to normalized data. The classification engine calculates the cognitive score of the EEG signals of the learner. Next, it extracts the feature sets from the normalized data as per the cognitive score. The feature set would mostly consist of time and cognitive scores. Then, the feature sets are taken in the ML algorithm considering the exploratory data analysis (EDA) of the normalized data. Lastly, the ML algorithm of the classification engine classifies the learner category as Beginner or Expert levels. 


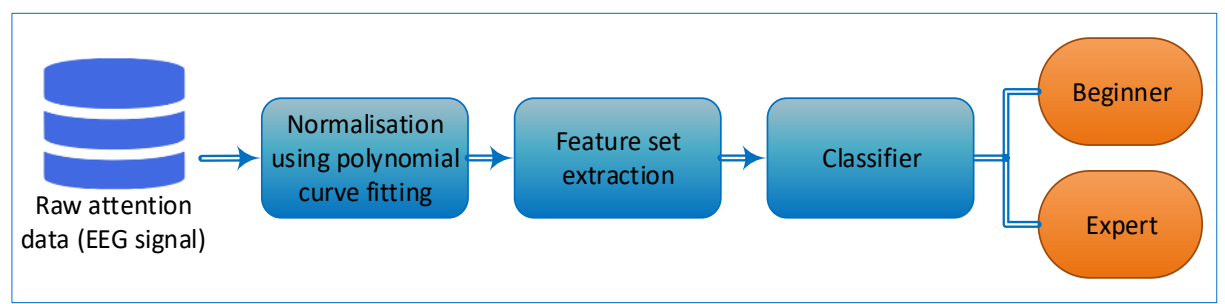

Figure 4. The workflow of the classification engine

\section{Experimental Results}

The present section presents the results of the study. The plots of attention level in cognitive state estimation are discussed, along with a discussion on classification. Ground truth on AI course taken by them is also collected. As shown in Figures 5 and 6, the number of undergraduate and postgraduate along with their knowledge in the field of AI. Out of 273 undergraduate students, 101 have basic concepts in the subject, and for 267 postgraduate students, 202 have prior AI knowledge. we will use this ground truth and verify from our framework.

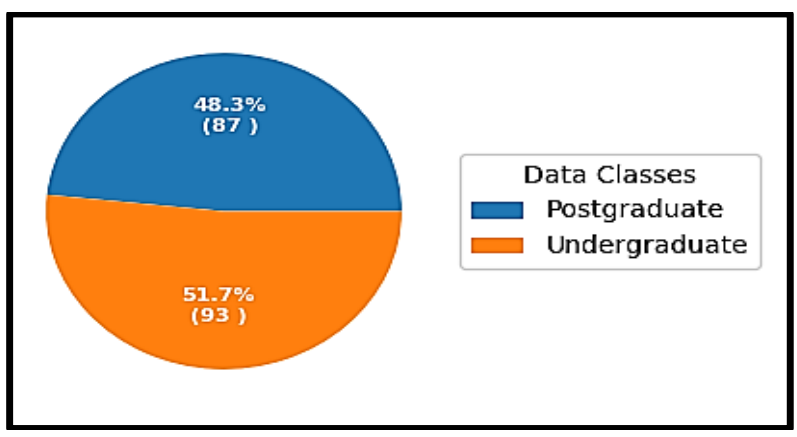

Figure 5. Data class

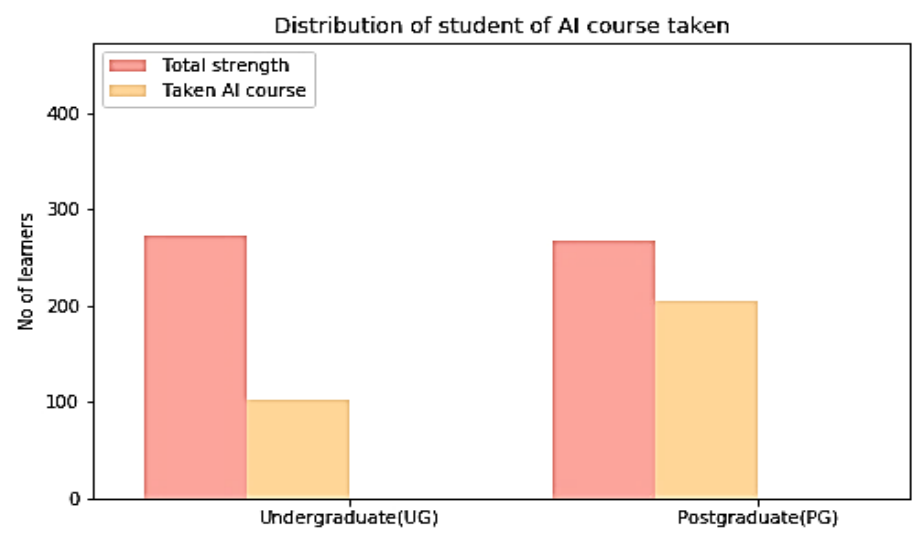

Figure 6. Learner distribution class 


\subsection{Plots of Attention Level in Cognitive State Estimation}

Currently, the attention levels of the students to define cognitive state changes are being dynamically investigated. This will ensure that information about the cognitive score and cognitive load for quick learner classification is gathered. For example, in Figure 7, the different attention levels of the raw dataset with respect to time are plotted. But, from the plot of Figure 7, cognitive score information cannot be obtained.

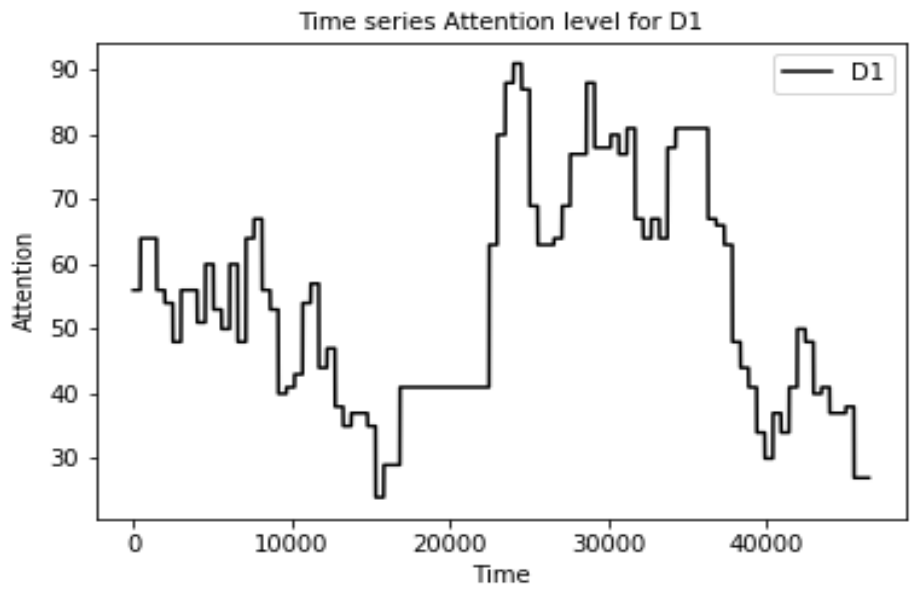

Figure 7. Raw data plot

Then, the mean value of the attention dataset is calculated and plotted in the raw plot of Figure 7. Figure 8 shows the raw plot and mean attention, but still, no information about the cognitive score can be obtained from the plot.

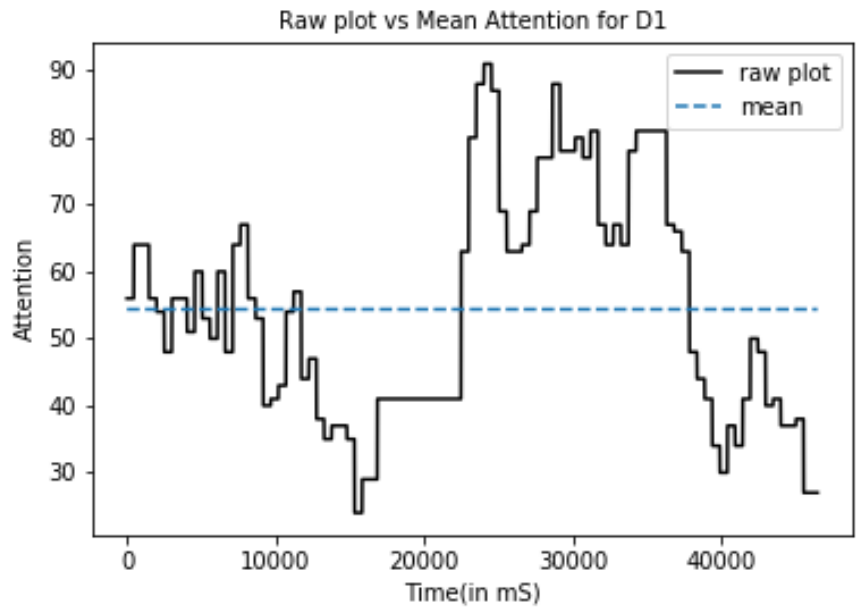

Figure 8. Raw data plot with mean attention

As no conclusions can be drawn from the raw plot and mean plot of the attention dataset, a polynomial plotting is needed. Hence, the curve fitting of degree 3 needs to be applied for getting 
information from the plot. For example, Figure 9 shows the fitting of a polynomial curve of normalized data of degree 3 for gathering information about the increase and decrease of attention levels. Attention level is high where the curve rises upwards, and attention level is low where the curve dips downwards.

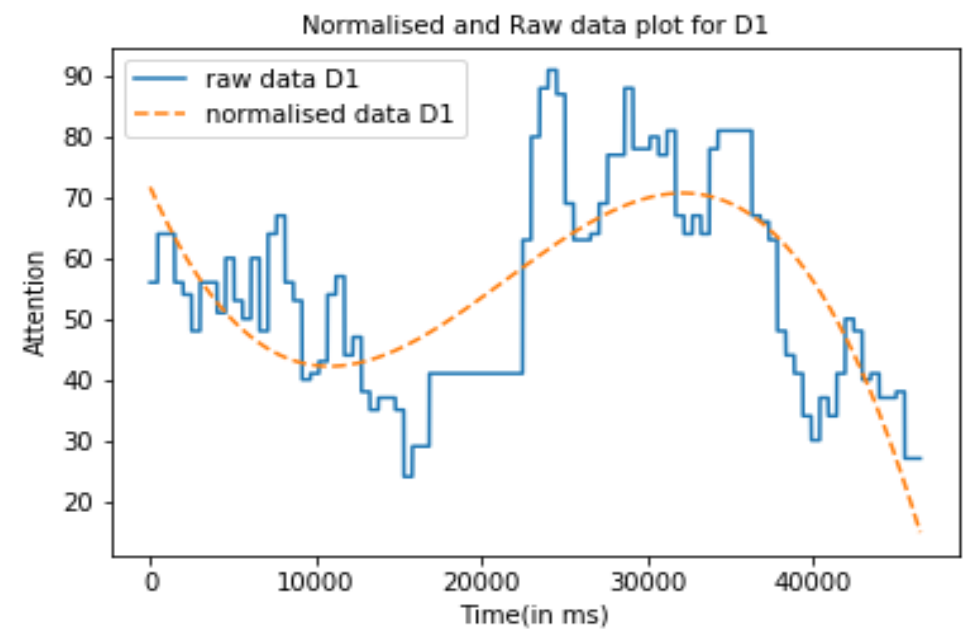

Figure 9. Raw data plot with curve fitting of degree 3

Next, the standard deviation of mean values is calculated as the standard deviation high and standard deviation low. Then, standard deviation high and standard deviation low curve is plotted into the raw data plot where standard deviation high $=$ mean $+($ standard deviation $/ 2)$ and standard deviation low $=$ mean - (standard deviation/2).

Figure 10 shows the application of the standard deviation method into a raw data plot for D2. Thus, maximum information can be derived between standard deviation high and standard deviation low curve. The outer part of this area is called an exceptional point.

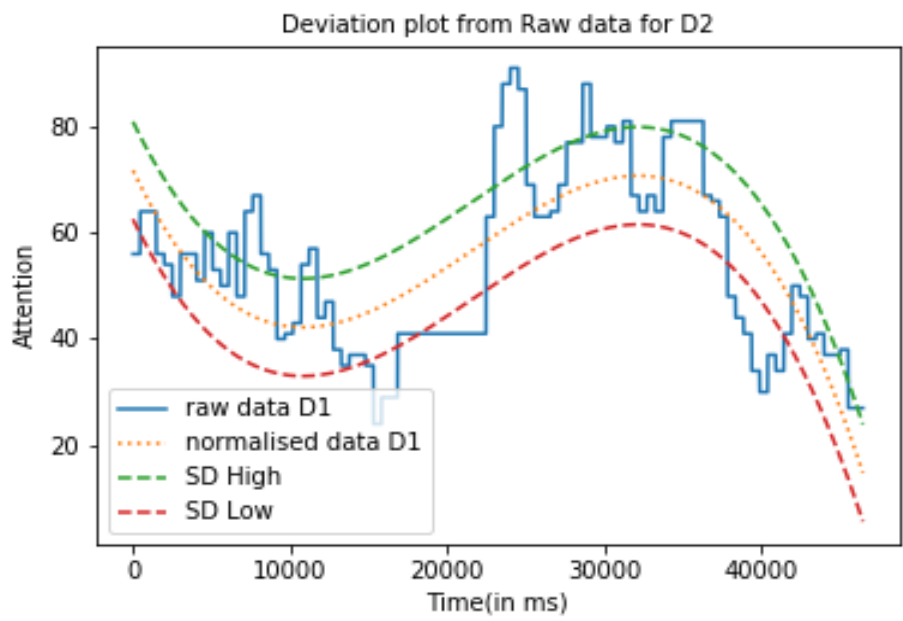

Figure 10. Derivation plot for raw data for D2 


\subsection{Classification}

Figure 11 shows the plot of the cognitive score of the dataset with respect to time. The plots are justified. Figure 11 is a pair plot of the cognitive score and time as a feature to distinguish between the master's students (1) which are represented by orange dots and the diploma students (0) as blue dots. Clearly, this shows that the machine can effectively distinguish between the two types of learners using the above two parameters. Also, the values on either side are actually correct which is not possible with a traditional approach as there can be a master's student without knowledge of a topic (here, artificial intelligence) who must be treated as a beginner, and the same is true for a diploma student having prior knowledge of the topic. So, this system can effectively classify them, not on a hypothesis, but with data.

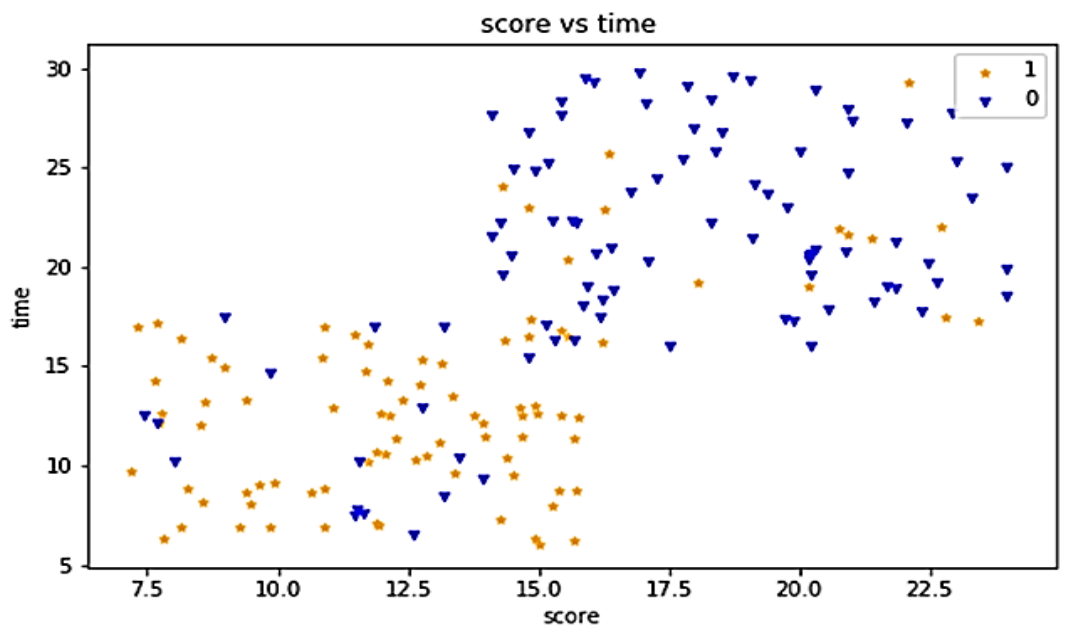

Figure 11. Variation of cognitive scores of the dataset with time

\section{Conclusions}

A conventional classroom, in which a teacher conducts a face-to-face interaction with students, enables the teacher to assess if students are focusing on the lecture-based on facial expressions and body movements. But then, where distance-learning or multimedia is concerned, a teacher faces more difficulty in assessing if students can understand and are attentive. Hence, this investigation used a new approach for analysing the learning process of students, thus enabling students, teachers, and other concerned persons to gauge if the students are attentive by employing technology. Such a method permits teachers to accordingly change the course content, improve student learning abilities, and bring back student attention when it wanders.

In this study, a system capable of monitoring the progress of students using brainwaves is developed in order to enable the dynamic adjustment of instructional methods and/or materials within a quick learning environment. It has been shown that the designed system gathers the EEG brainwaves, assesses the student's instantaneous attention level, transfers this data to an android app, and transmits the information to the server. The attention readings of the various learners (which include both those at a masters and diploma level) are monitored continuously. Certain parameters are decided to evaluate the total cognitive load of the presented document. The proposed algorithm calculates the cognitive load. 
The spectral response is used in various channels to derive correlations with higher test scores. Using the feature data sets, a classification engine for recommending different levels of quick learners is proposed. It has been found that the cognitive load is dependent on the learning resource and varies with the documents.

\section{References}

[1] G. Secundo, A. Margherita, G. Elia and G. Passiante, "Intangible assets in higher education and research: Mission, performance or both?," Journal of Intellectual Capital, vol. 11, no. 2, pp. 140-157, 2010.

[2] D. Millett, "Hans Berger: From Psychic Energy to the EEG," Perspectives in Biology and Medicine, pp. 522-542, 2001.

[3] B. Duch, D. \&. W. Allen and I. H. B, "http://teaching.polyu.edu.pdf," 2009. [Online]. Available: http://teaching.polyu.edu.pdf. [Accessed 1110 2019].

[4] B. Trilling and P. Hood, "Learning, technology, and education reform in the Knowledge Age or "We're Wired, Webbed, and Windowed, Now What?"," Educational Technology, vol. 39, no. 3, pp. 5-18, 1999.

[5] S. Pal, M. Mukhopadhyay, P. K. D. Pramanik and P. Choudhury, "Assessing the Learning Difficulty of Text-Based Learning Materials," in 7th International Conference on Frontiers of Intelligent Computing: Theory and Application (FICTA 2018), Da Nang city, Viet Nam, November, 2018.

[6] S. Pal, P. K. D. Pramanik and P. Choudhury, "A Step Towards Smart Learning: Designing an Interactive Video-Based M-Learning System for Educational Institutes," International Journal of Web-Based Learning and Teaching Technologies, vol. 14, no. 4, pp. 26-48, 2019.

[7] NeuroSky, Inc, "Brain Wave Signal (EEG) of NeuroSky, Inc.," 1512 2009. [Online]. Available: http://www.frontiernerds.com/files/neurosky-vs-medical-eeg.pdf. [Accessed 1010 2019].

[8] Z. D. Dragoljub Gajic and F. G. Stefano Di Gennaro, "Classification Of EEG Signals For Detection Of Epileptic Seizures Based On Wavelets And Statistical Pattern Recognition," Biomedical Engineering: Applications, Basis and Communications, vol. 26, no. 2, 2014.

[9] R. O'Reilly, "Classroom Climate and Achievement in Secondary School Mathematics Classes," Alberta Journal of Educational Research, vol. 21, no. 4, pp. 241-248, 1975.

[10] T. Kaur, "Characteristic behavioural and environmental correlates of academic achievement of over and under achievers at different levels of intelligence ix class pupils in Urban areas of the Panjab," Department of Education, Panjab University, 1975.

[11] N. Hussain, B. Nawaz, S. Nasir, N. Kiani and M. Hussain, "Positive Teacher-Student Relationship and Teachers Experience - A Teacher's Perspective," Global Journal of Management and Business Research, vol. 13, no. 3, pp. 1-5, 2013.

[12] Pacific Policy Research Centre, "21st century skills for students and teachers. Honolulu: Kamehameha Schools," Research \& Evaluation Division, 2010.

[13] G. Pfurtscheller and W. Klimesch, "Event-Related Synchronization and Desynchronization of Alpha and Beta Waves in a Cognitive Task," in Induced Rhythms in the Brain, E. Başar and T. Bullock, Eds., Birkhäuser, Boston, MA, 1992, pp. 117-128. 
[14] A. Bose, S. S. Roy, V. E. Balas and P. Samui, "Deep Learning for Brain Computer Interfaces," in Handbook of Deep Learning Applications, V. E. Balas, S. S. Roy, D. Sharma and P. Samui, Eds., Cham, Springer, 2019, pp. 333-344.

[15] M. A.-K. Nafea and F. Harun, "Brainwave-Controlled System for Smart Home Applications," in 2nd International Conference on BioSignal Analysis, Processing and Systems (ICBAPS), 2018.

[16] A. Choppin, "EEG-based human interface for disabled individuals: Emotion expression with neural networks," in Master's thesis, Tokyo, Tokyo Institure of Technology, 2000.

[17] T. Musha, Y. Terasaki, H. A. Haque and G. A. Ivamitsky, "Feature extraction from EEGs associated with emotions," Artificial Life and Robotics, vol. 1, no. 1, pp. 15-19, 1975.

[18] A. Heraz, R. Razaki and C. Frasson, "Using machine learning to predict learner emotional state from brainwaves," in Seventh IEEE International Conference on Advanced Learning Technologies (ICALT 2007), 2007.

[19] G. Chanel, J. J. Kierkels, M. Soleymani and T. Pun, "Short-term emotion assessment in a recall paradigm," International Journal of Human-Computer Studies, vol. 67, no. 8, pp. 607-627, 2009.

[20] Q. Zhang and M. Lee, "Analysis of positive and negative emotions in natural scene using brain activity and GIST," Neurocomputing, vol. 72, no. 4-6, pp. 1302-1306, 2009.

[21] Y. P. Lin, C. H. Wang, T. P. Jung, T. L. Wu, S. K. Jeng, J. R. Duann and J. H. Chen, "EEGbased emotion recognition in music listening," IEEE Transactions on Biomedical Engineering, vol. 57, no. 7, pp. 1798-1806, 2010. 